\title{
Etude sur le salaire horaire: des résultats stables
}

\section{Beatrice Brunnera, Esther Kraft ${ }^{\text {b }}$}

${ }^{a}$ WIG/ZHAW, responsable suppléante en recherche économique sur les soins, Winterthour; ${ }^{b}$ FMH, cheffe de la division Données, démographie et qualité

\section{Introduction}

Début 2019, la Haute école des sciences appliquées de Zurich (ZHAW) a publié une étude commanditée par la FMH, qui comparait le salaire horaire des médecins à celui d'autres professions académiques [1]. L'édition révisée de cette étude est parue dernièrement [2]. Pour la FMH, la transparence et la fiabilité des données constituent le fondement d'une discussion objective, basée sur des faits et orientée sur la recherche de solutions concernant le revenu des médecins. Aussi, l'article qui suit présente les nouveaux résultats en se référant à l'étude MAS (Medical Ambulatory - Structure) de l'Office fédéral de la statistique (OFS).

\section{L'étude doit "comparer ce qui est comparable»}

L'objectif de l'étude est de calculer le salaire horaire effectif des médecins suisses en tenant compte de leur temps de travail hebdomadaire, du nombre de jours

L'objectif de l'étude est de calculer le salaire horaire effectif des médecins suisses.

de vacances, et des jours fériés cantonaux (tombant sur un jour de semaine). Sur la base des même données, les auteurs calculent également le salaire horaire d'autres groupes professionnels pour le comparer à celui des médecins.

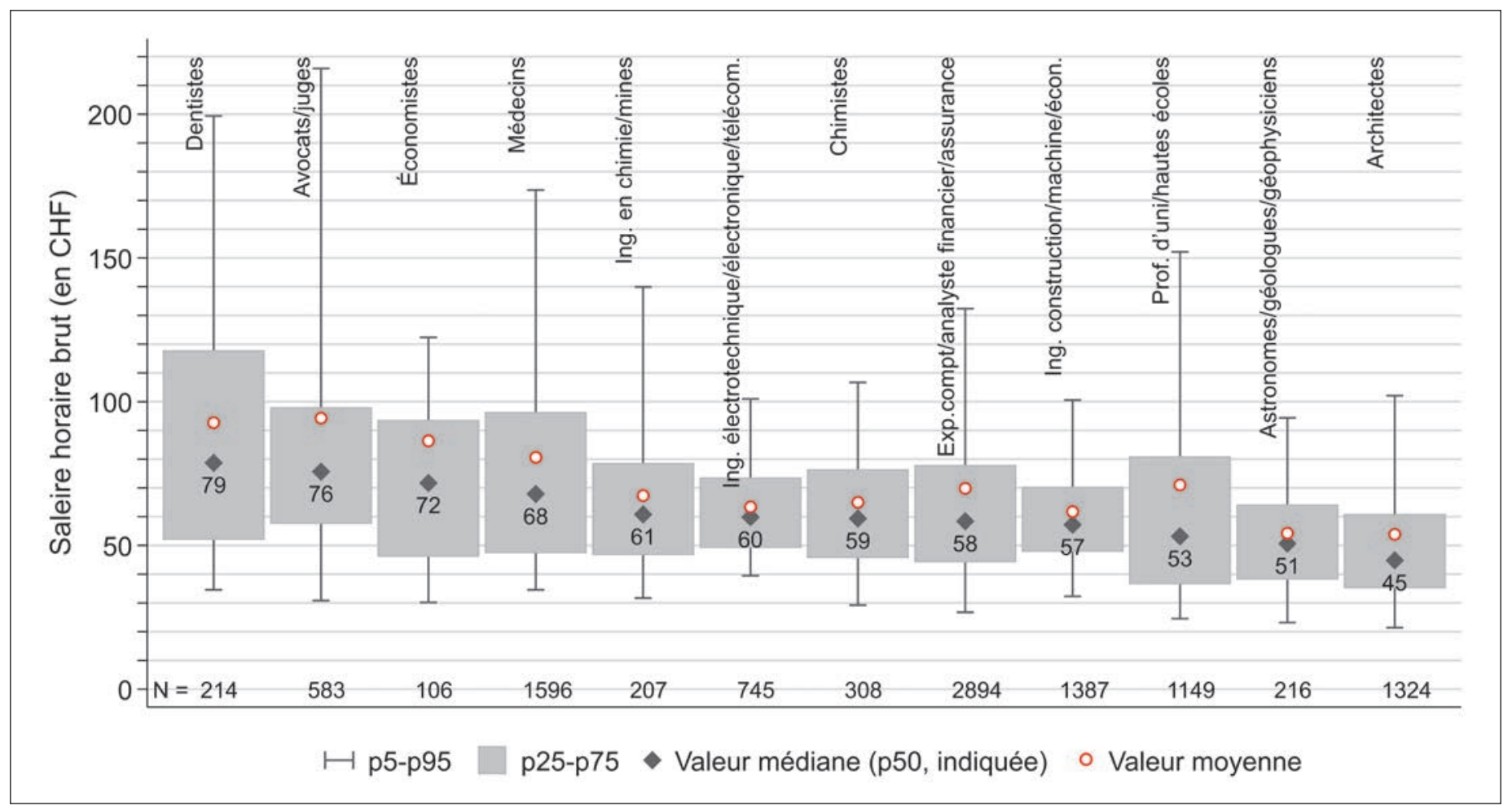

Figure 1: Revenu horaire brut pour plusieurs professions académiques (2014-2017). 


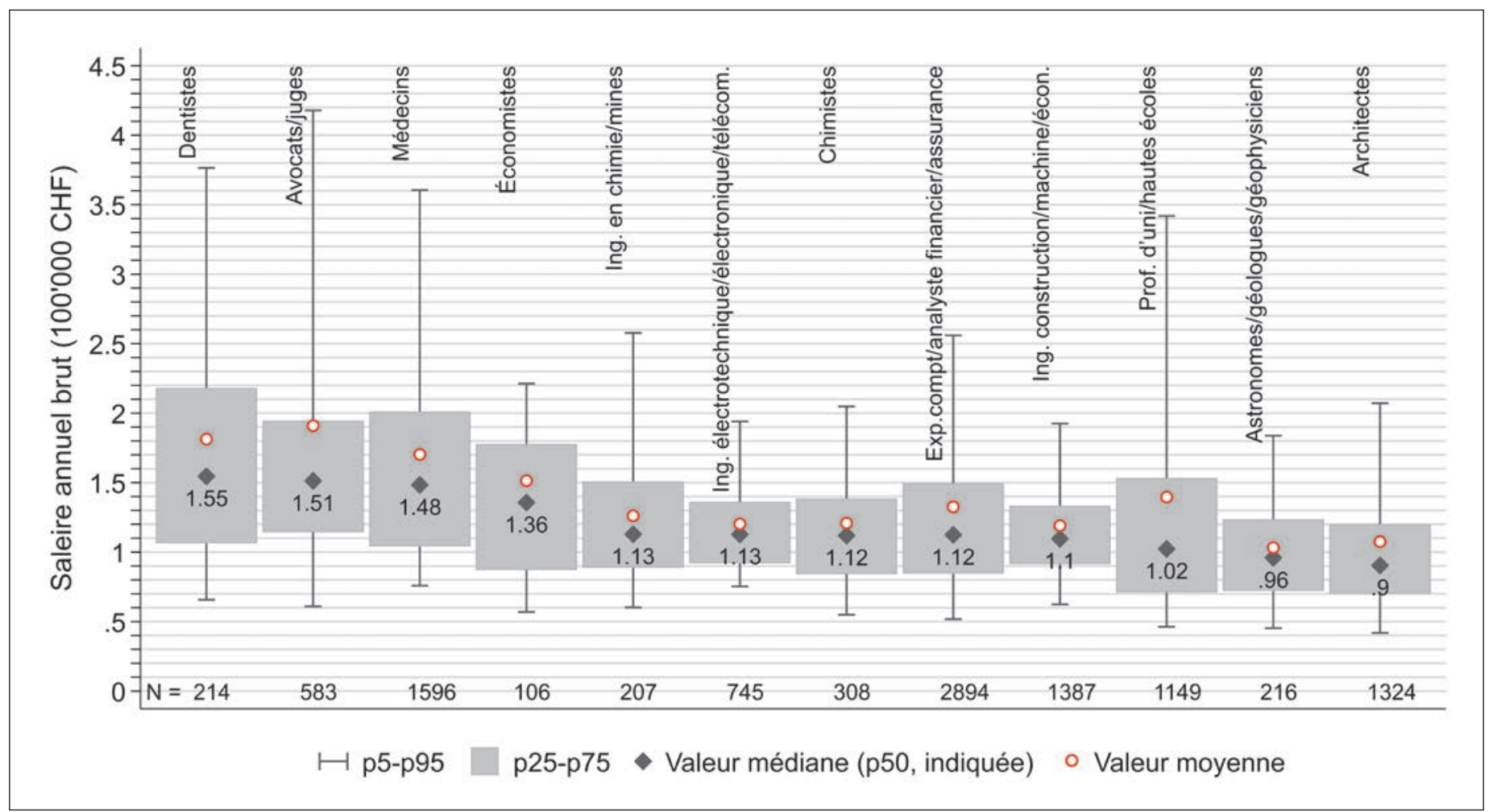

Figure 2: Revenu annuel brut pour plusieurs professions académiques (2014-2017).

Les résultats de la première édition sont parus en janvier 2019 [1]. Dans la deuxième édition [2], les médecins sont d'une part identifiés de manière plus complète, ce qui améliore la représentativité des données, et, d'autre part, les salaires horaires sont reproduits de manière plus précise.

\section{L'ESPA est une enquête par échantillonnage menée chaque année par l'OFS auprès de la population.}

L'étude s'appuie sur l'enquête suisse sur la population active (ESPA), réalisée chaque année par l'OFS auprès de quelque 40000 actifs. L'ESPA comprend entre autres des informations sur le métier, le statut professionnel, le secteur d'activité de l'entreprise, le salaire, les jours de vacances pris ainsi que le nombre d'heures hebdomadaires effectivement travaillées. Nous remercions l'OFS pour les remarques techniques faites dans le cadre de la première version de l'étude et prises en compte dans la deuxième édition.

\section{Résultats de l'étude sur le salaire horaire}

Comme le montre la figure 2, le revenu annuel brut médian des médecins compris dans l'ESPA s'élevait à 148000 francs entre 2014 et 2017. Ils se placent donc troisièmes parmi les catégories professionnelles considérées. Le salaire médian le plus élevé est celui des dentistes, à 155000 francs par an, suivis des avocats et des juges, à 151000 francs par an. Les différences s'accentuent lorsqu'on compare les salaires horaires. Avec un salaire horaire médian de 68 francs, les médecins se placent en quatrième position (figure 1).

L'analyse de représentativité ayant montré que les jeunes médecins (surtout les moins de 35 ans) sont surreprésentés dans l'ESPA, et que les salaires des médecins sont donc sous-évalués, le même comparatif a été établi une seconde fois pour les 35 ans et plus. Lâge moyen d'acquisition du titre de spécialiste étant d'environ 36 ans, la plupart des médecins-assistants

Avec un salaire horaire médian de 78 francs, les médecins se placent à nouveau quatrièmes.

sont donc exclus de l'étude. La médiane des revenus annuels bruts standardisés des médecins de 35 ans ou plus s'élève alors à 168000 francs (figure 4). Les médecins se placent dès lors premiers, juste avant les dentistes (166000 francs/an), les avocats et les juges (163000) et les économistes (162000). Le classement est tout autre si l'on considère les salaires horaires. Avec un salaire horaire médian de 78 francs, les médecins se placent à nouveau quatrièmes, derrière les 
dentistes, à 88 francs/h, les économistes, à 86 francs/h, et les avocats et les juges, à 82 francs/h (figure 3). Les deux comparaisons montrent que le revenu annuel relativement élevé des médecins par rapport aux autres groupes de professions de niveau académique s'explique notamment par leur nombre élevé d'heures de travail hebdomadaires. Le temps de travail hebdomadaire médian des médecins de 35 ans ou plus s'inscrit à 49,5 heures, un chiffre nettement plus élevé que pour les autres professions.

\section{Résultats du relevé MAS 2017}

Le 24 octobre 2019, l'OFS a publié les résultats du relevé MAS concernant les données structurelles des cabinets médicaux et des centres ambulatoires pour l'année 2017 [3]. Effectué sur la base des art. 23 et 59a de la loi sur l'assurance-maladie (LAMal) et de la loi

\section{Le relevé MAS fait état d'un revenu net de 154000 francs pour les médecins en cabinet individuel.}

sur la statistique fédérale (LSF), le relevé a servi à des fins d'analyse statistique, mais également de surveillance. Par rapport au premier relevé, le taux de contact ainsi que le taux de réponses se sont nettement améliorés, passant respectivement de 70 à $82 \%$ et de 52 à 68\%. Le relevé MAS 2017 a ainsi permis une analyse fondée de la situation financière des cabinets médicaux et des centres ambulatoires en Suisse. Pour

En moyenne, 85\% des recettes encaissées par les cabinets proviennent de l'assurance obligatoire des soins.

les médecins en cabinet individuel, l'analyse révèle des recettes totales médianes de 428000 francs et des charges totales médianes de 263000 francs. Le revenu net médian s'inscrit ainsi à 154000 francs en 2017. En moyenne, $85 \%$ des recettes encaissées par les cabinets en 2017 proviennent de l'assurance obligatoire des soins [3]. Le relevé par ailleurs MAS 2017 révèle un temps de travail hebdomadaire moyen de 40 heures pour les médecins, ce qui correspond à un taux d'occupation de $75 \%$. Cela signifie que les médecins travaillent un quart de plus que la moyenne suisse, des chiffres confirmés par l'étude examinant les salaires horaires, qui montre que les médecins travaillent un peu plus de $18 \%$ plus longtemps que les autres professions de niveau universitaire. En tenant compte en outre des jours de vacances, répertoriés dans l'étude sur les salaires horaires, les données du relevé MAS 2017 permettent de conclure à un salaire horaire net d'environ 84 francs pour les médecins en cabinet individuel.

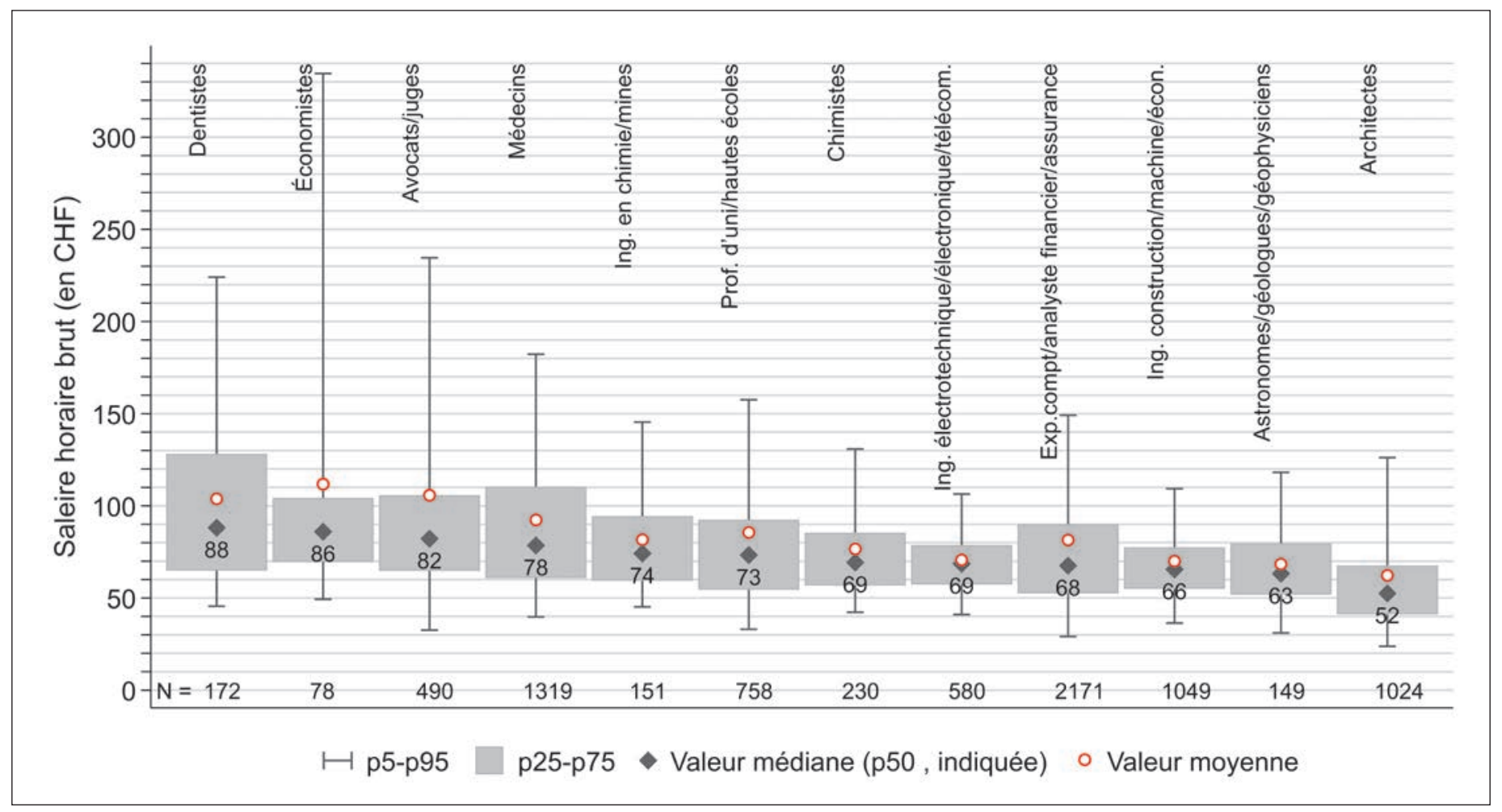

Figure 3: Revenu horaire brut pour plusieurs professions académiques (2014-2017), à partir de 35 ans. 


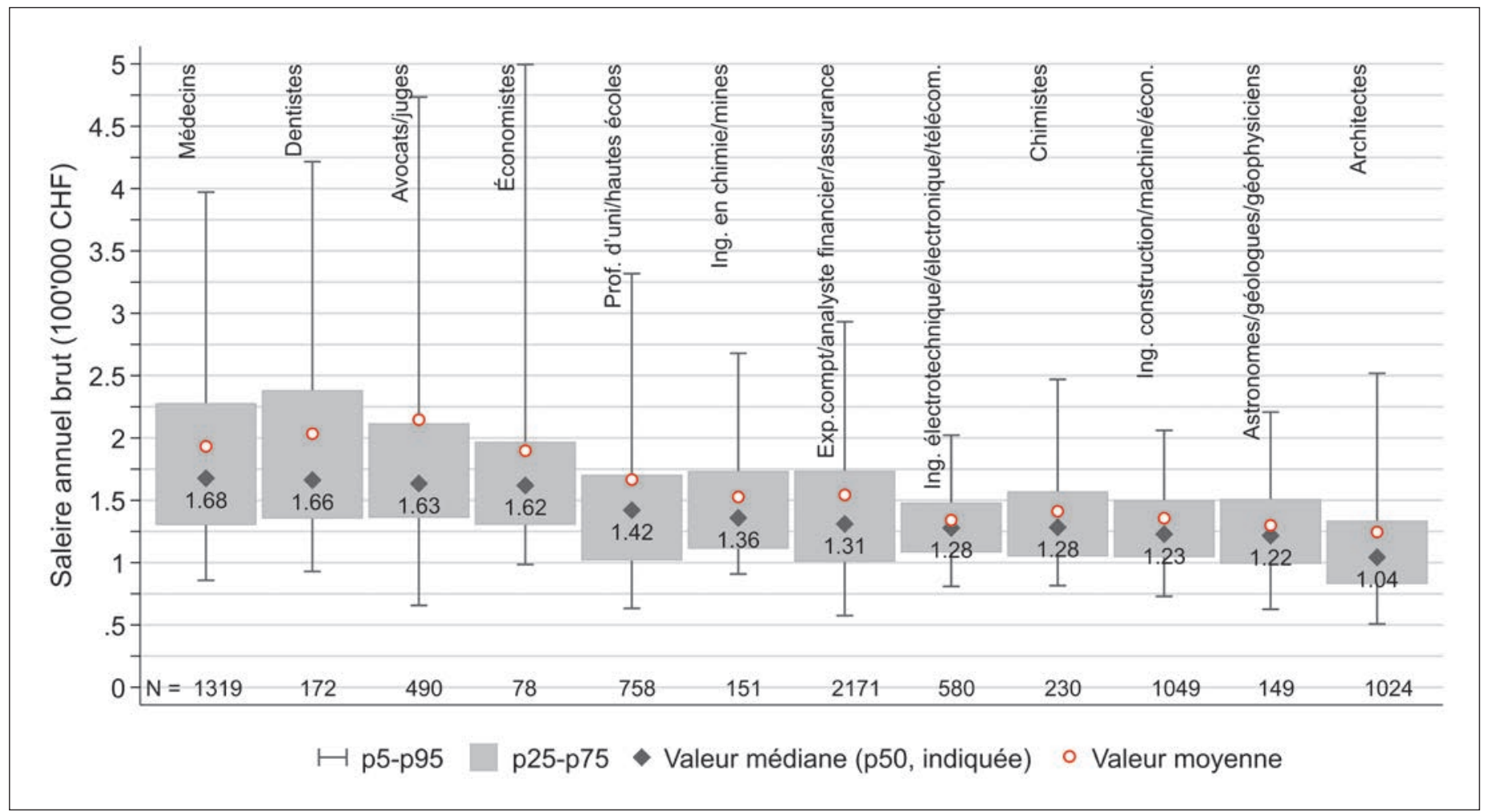

Figure 4: Revenu annuel brut pour plusieurs professions académiques (2014-2017), à partir de 35 ans.

Beatrice Brunner WIG / ZHAW

Gertrudstrasse 15

CH-8401 Winterthour

beatrice.brunner[at]zhaw.ch

Esther Kraft

FMH

Elfenstrasse 18

CH-3000 Berne 15

esther.kraft[at]fmh.ch

\section{Conclusions et perspectives}

L'étude révisée sur les salaires horaires calcule et compare les salaires horaires des médecins à ceux d'autres groupes professionnels en Suisse. Elle opère sur un ensemble statistiquement clairement délimité (ESPA), et permet de comparer ce qui est comparable.

Le relevé MAS 2017 de l'OFS recense désormais aussi les heures de travail hebdomadaires et permet de valider les résultats de l'étude sur le salaire horaire. Comme le révèle une analyse sommaire, les résultats sont effectivement comparables et peuvent servir de base à une discussion objective, basée sur des faits et orientée sur la recherche de solutions.
Références

1 Brunner B, Kraft E. Comparaison entre les médecins et d'autres groupes professionnels. Etude sur le salaire horaire. Bull Med Suisses. 2019;100(6):158-9.

2 Etude Brunner: https://digitalcollection.zhaw.ch/ bitstream/11475/18477/1/2019_Stundenlohn\%20Aerzte_ Brunner.pdf

3 MAS 2017 - résultats, site Internet de l'OFs: https://www.bfs. admin.ch/bfs/fr/home/statistiques/sante/systeme-sante/ cabinets-medicaux htm

\section{Crédit photo}

WIG/ZHAW 